\title{
Going the distance with success: An attempted argument that less is not always more
}

\author{
Jonathan W. Haft, MD
}

\author{
From the Department of Cardiac Surgery, University of Michigan, Ann Arbor, Mich. \\ Disclosures: Author has nothing to disclose with regard to commercial support. \\ Received for publication Oct 8, 2015; accepted for publication Oct 17, 2015; available ahead of print Nov 13, \\ 2015 \\ Address for reprints: Jonathan W. Haft, MD, Department of Cardiac Surgery, University of Michigan, $1500 \mathrm{E}$ \\ Medical Center Dr, 5144 CVC/SPC 5864, Ann Arbor, MI 48109-0344 (E-mail: haft@umich.edu). \\ J Thorac Cardiovasc Surg 2016;151:528-9 \\ $0022-5223 / \$ 36.00$ \\ Copyright (c) 2016 by The American Association for Thoracic Surgery \\ http://dx.doi.org/10.1016/j.jtcvs.2015.10.041
}

Advanced heart failure is increasingly treated with durable mechanical circulatory support using continuous flow left ventricular assist devices (LVADs). Right ventricular (RV) dysfunction can affect the effectiveness of this therapy, both in terms of short-term morbidity and in terms of long-term survival and adverse events. Because of the mandatory coupling of the 2 ventricles, RV dysfunction is extremely common in patients with end-stage heart failure and is often associated with geometric changes resulting in tricuspid valve insufficiency. In addition, many of these patients have internal defibrillators traversing the tricuspid apparatus, which can distort the leaflets and magnify the severity of tricuspid regurgitation (TR). Left untreated, severe TR will exacerbate RV volume overload and can lead to persistent heart failure symptoms, progressive renal and hepatic dysfunction, and reduced survival. LVAD implantation unloads the left ventricle and reduces both pulmonary arterial pressures and pulmonary vascular resistance. It remains unclear whether this right-sided afterload reduction can resolve TR and prevent RV failure. There is not uniform agreement in the LVAD community regarding the appropriate trigger to add simultaneous tricuspid valve repair or replacement during LVAD implantation.

The report in this issue of the Journal from Han and colleagues from Columbia University describes a singleinstitutional retrospective analysis of patients implanted with continuous flow LVADs (90\% HeartMate II, 10\% HeartWare and others), comparing characteristics and outcomes of those with and without tricuspid intervention (mostly ring annuloplasty). The 2 cohorts were surprisingly quite similar, with the exception of slightly higher central venous pressure (CVP; $12 \mathrm{~mm} \mathrm{Hg}$ vs $11 \mathrm{~mm} \mathrm{Hg}$ ), higher ratio of CVP to wedge pressure, more anemia (hematocrit $33 \%$ vs $35 \%$ ), higher bilirubin ( 1.8 vs $1.4 \mathrm{mg} / \mathrm{dL}$ ), and of course worse grade of TR (mild or less, $1 \%$ vs $76 \%$; more than moderate, $76 \%$ vs $3 \%$ ). The operations were longer in those that had a tricuspid procedure (cardiopulmonary bypass time 136 vs 84 minutes). There was an

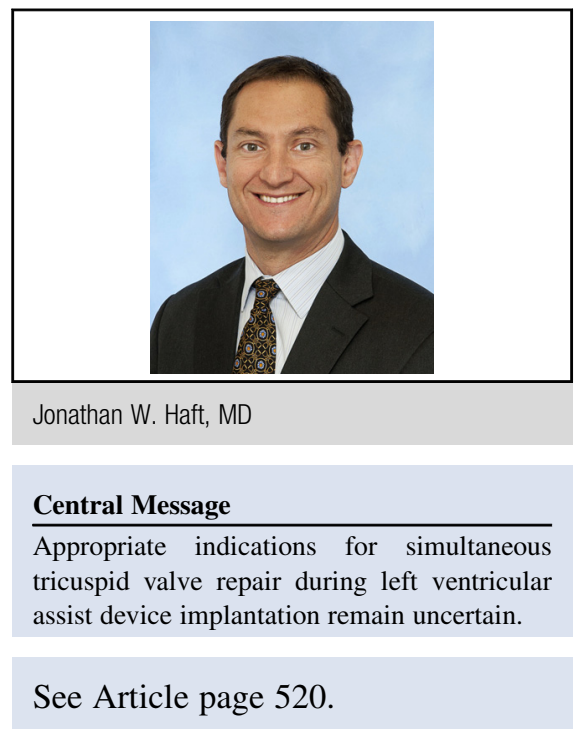

associated increased use of platelet transfusions (14 vs 12 units), and there were more patients who required return to the operating room for bleeding in the tricuspid repair group ( $28 \%$ vs $17 \%)$. Critical outcomes were similar between the groups, with an in-hospital mortality of $7 \%$ and a hospital stay of 36 days. There was no difference in overall survival, which was $74 \%$ at 2 years for those that remained on LVAD support. Overall rates of hospital readmissions were similar, including the rate of hospital readmission for $\mathrm{RV}$ failure. There were important differences in survival to 6 months when broken down by severity of TR, with survival worse as the grade of TR increased. For those patients with moderate TR, there appeared to be a survival advantage when the tricuspid was repaired, although this benefit did not achieve statistical significance. Tricuspid intervention was largely successful, with the vast majority of patients having less than moderate TR according to echocardiographic follow-up to 24 months. Tricuspid valve intervention significantly reduced the likelihood of development of moderate or worse TR. Han and colleagues conclude that tricuspid valve intervention is safe, that tricuspid repair is durable in its effectiveness, and that tricuspid repair may improve long-term survival in patients with at least moderate TR.

There are likely 2 camps with respect to functional TR in the LVAD community: those who aggressively address the tricuspid simultaneously and those who do not. I suspect that this well-written report is unlikely to change substantially the attitudes of either party's constituents. Those in 
favor of an aggressive repair strategy will point out that patients who received tricuspid intervention were sicker, as evidenced by their higher bilirubin, CVP to wedge pressure ratio, and greater anemia. Despite those differences, both perioperative and long-term survivals were identical, suggesting that tricuspid repair may have affected these outcomes. Proponents of tricuspid repair would also argue that survival of patients with moderate TR left untreated was worse than that of those who underwent tricuspid intervention, although that comparison did not achieve statistical significance because of the insufficient size of the cohorts. An argument could also be made that the rates of recurrent hospitalization for RV failure were similar, despite the higher preoperative prevalence of severe RV failure, both echocardiographically and clinically (higher CVP and bilirubin). Tricuspid repair was also durable, with few cases of recurrent significant TR.

Despite these important findings, I am uncertain whether surgeons opposed to an aggressive tricuspid strategy will find these data convincing enough to change their practice. There were no significant differences in late complications directly attributed to RV failure or TR. The frequency of development of late severe TR was low in the cohort of patients who did not undergo intervention. In addition, simultaneous tricuspid repair was associated with longer cardiopulmonary bypass time, greater need for blood transfusions, and a higher rate of return to the operating room for postoperative bleeding. In addition to the potential harm that comes from adding time and complexity to the operation, simultaneous tricuspid intervention impacts the potential for a minimally invasive approach to LVAD implantation either through a left thoracotomy alone or through a minithoracotomy coupled with partial upper sternotomy. This will become particularly important as newer and smaller LVADs, which are more conducive to minimally invasive techniques, become available.

This report from the Columbia group is among the largest single series of LVAD recipients with excellent outcomes and robust statistical analysis. The investigation is limited, however, by an insufficient sample size in a control group of patients with at least moderate TR. Furthermore, duration of follow-up is affected by censorship at the time of heart transplant, potentially underestimating the impact of untreated TR. Although Han and colleagues have convincingly demonstrated admirable long-term survival as well as durable effectiveness of tricuspid repair, we need to understand better the consequences of leaving significant TR alone. A compelling argument likely cannot be made from a single institution's report, in which decision making was necessarily influenced by inherent biases. These questions need to be answered from prospective trials or potentially from highly granular multicenter registries representing programs with disparate approaches to TR. The important question of simultaneous tricuspid repair may be analogous to other scenarios in cardiac surgery in which minimally invasive strategies mandate more selective interventions. We need to understand better when doing less may actually be more. 